Discussion Paper No. 601

\title{
LEARNING, LIQUIDITY PREFERENCE, AND BUSINESS CYCLE
}

Ryo Horii and Yoshiyasu Ono

March 2004

The Institute of Social and Economic Research Osaka University

6-1 Mihogaoka, Ibaraki, Osaka 567-0047, Japan 


\title{
Learning, Liquidity Preference, and Business Cycle*
}

\author{
Ryo Horii ${ }^{\dagger}$ and Yoshiyasu Ono
}

February 2004

\begin{abstract}
This paper examines a mechanism of liquidity-preference fluctuations caused by changes in people's belief about a random liquidity shock. When observing the shock, they rationally update their belief so that the shock probability is higher; consequently they raise liquidity preference and reduce consumption. As the period without the shock lasts, they become more optimistic so that they gradually lower liquidity preference and increase consumption. The recovery pattern depends on the realized frequency of the shock: when the shock occurs many times in succession, the consumption recovery is first slow, gradually accelerates and eventually slows down, tracing an 'S'-shaped curve.
\end{abstract}

JEL Classifications: D83, E41, E32

Keywords: Bayesian Learning, Liquidity Preference, Precautionary Motive, Business Cycle, Markov Switching

${ }^{*}$ The authors are grateful to Kazuo Mino, Akihisa Shibata, and seminar participants at the University of Tokyo for their helpful comments and suggestions.

${ }^{\dagger}$ Graduate School of Economics, Osaka University, 1-7 Machikaneyama, Toyonaka, Osaka 5600043, JAPAN; E-mail: horii@econ.osaka-u.ac.jp

${ }_{\ddagger}^{\ddagger}$ Institute of Social and Economic Research, Osaka University, 6-1, Mihogaoka, Ibaraki-shi, Osaka, 567-0047, JAPAN; E-mail: ono@iser.osaka-u.ac.jp 


\section{Introduction}

Why do people hold money although money does not generate interest earnings? In the literature a wide variety of reasons for liquidity holding have been discussed, such as the transaction motive, the speculative motive, status preference, etc. This paper focuses on the precautionary motive. People hold money since they prepare for a possible aggregate liquidity shock, such as bank runs or sudden malfunction of the financial system. By observing whether or not such a shock actually occurs at each point in time they rationally update the subjective probability of the shock, causing their liquidity preference to fluctuate. The fluctuation in turn affects the optimal time path of consumption and generates a demand-driven business cycle. This paper examines such a business-cycle mechanism.

If transactions are properly settled by checks or credit cards, people need not hold money stock. However, if a financial crisis occurs and checks or credit cards do not properly work, holding money stock yields an explicit benefit. Bank runs can also be regarded as another example of such liquidity shocks. As long as banks normally operate, people need not hold money since they can easily withdraw money whenever they buy something. Thus, at normal times holding money does not directly benefit people. If a bank run occurs, however, people cannot withdraw money so that they have to use the money that they hold. On this occasion holding money generates utility.

Since such crises cannot exactly be anticipated, people hold money even when it is unnecessary. Such liquidity preference depends on how exactly people know about the probability of the shock at each point in time. If they know the exact probability and it does not change, the expected gain of holding money is constant over time. Thus, they behave as if they had a deterministic utility function of money as originally assumed in Sidrauski (1967). If they do not know the exact probability, whether the shock occurs or not today conveys some information for people to guess 
the probability of the shock tomorrow. More precisely, when people observe the shock, they update their subjective probability for meeting with it again and raise liquidity preference. If the shock does not occur for a while, they gradually reduce the probability and lower liquidity preference.

In order to highlight such a fluctuation in liquidity preference we impose a rather extreme assumption: people receive utility from holding money only when the liquidity shock occurs. We also assume that there are two states with different probabilities of the shock and that people do not know which state they are in. The shock follows a Poisson process in each state. By observing whether or not the shock occurs at each point in time people rationally update the subjective probability using Bayes' law and revise the time paths of consumption and money holding. If nominal wage adjustment is sluggish in this setting, employment also fluctuates over time as liquidity preference varies. In this way we obtain a demand-driven business cycle.

In the literature of business cycles, there are a few attempts to explain cyclical movements of macroeconomic variables by combining unobservable regime changes and Bayesian updating agents. ${ }^{1}$ Chalkley and Lee (1998) and Potter (2000) examine how agents react to unobservable changes in an investment opportunity. Andolfatto and Gomme (2003) and Sill and Wrase (1999) focus on the situation where monetary policy, which is not directly observable, periodically switches between low and high monetary growth. In these models time is discrete and in each period agents receive a noisy signal with respect to the current state. Since the signal is noisy, agents slowly change their belief, making the effect of a regime change more persistent than in the case where the state is perfectly observable.

Besides the difference in motivation, our specification differs from them in that agents receive a signal (namely, observe the liquidity shock) only at sporadic points in

\footnotetext{
${ }^{1}$ Hamilton (1989) presents a different but somewhat related approach when viewing business cycles as regime changes. He assumes that it is not agents but econometricians that cannot directly observe regime changes, and develops a procedure to estimate the timing of regime changes.
} 
continuous time and form their belief depending upon when and how frequently the shock occurs. ${ }^{2}$ As long as the liquidity shock occurs sparsely in time, people view the shock as a mere accident. Consumption decreases only by a small amount and then quickly recovers. However, when the shock occurs many times for a short while, people are convinced that they are in the more dangerous state and significantly reduce consumption. In this case, the recovery process takes a long time because a period without the shock reveals information about the state little by little and thus people gradually lower liquidity preference. The recovery of consumption is then found to be initially slow, gradually accelerates and again slows down, tracing an 'S'-shaped curve. ${ }^{3}$

After modelling the belief-updating behavior of households in the next section, we examine liquidity-preference fluctuations and the optimal consumption behavior in section 3. Section 4 derives the existence, uniqueness and other properties of the stationary equilibrium path and presents the cyclical movements of the belief and consumption. Section 5 summarizes and concludes.

\section{Liquidity Shock and Bayesian Learning}

We use a continuous-time model in which a representative household faces an aggregate liquidity shock that follows an exogenous Poisson process. Liquidity holding generates utility when the shock actually occurs, but does not while the shock does

\footnotetext{
${ }^{2}$ There are some continuous-time models in which agents update their belief based on discrete signals although the unobservable state is time invariant. Zeira (1999) investigates bubbles in the stock market in which agents know that bubbles will crash some day but do not know exactly when. Driffill and Miller (1993) examine the inertia of the inflation rate under sluggish nominal price adjustment when agents are learning about the probability of sporadic exchange-rate realignments. In these models, however, uncertainty vanishes in the long run and recurrent cycles are not generated.

${ }^{3}$ This mechanism provides a possible explanation of why it took so long a time for consumer confidence in Japan to recover after experiencing a succession of bank failures in the 1990s.
} 
not occur. Since when the shock occurs cannot exactly be anticipated, even during the period without it the household holds liquidity so as to prepare for it.

There are two underlying states with different probabilities of the shock, called states $\mathrm{H}$ and $\mathrm{L}$. In state $i \in\{H, L\}$ the shock occurs with probability $\theta^{i}$ per unit time, where $\theta^{H}>\theta^{L}>0$. The household cannot directly observe the current state but knows that the state evolves according to a Markov process: state $\mathrm{H}$ changes to state L with Poisson probability $p^{H}$ per unit time whereas state L changes to state $\mathrm{H}$ with probability $p^{L}$. We assume that the shock occurs much more frequently in state $\mathrm{H}$ than in state $\mathrm{L}$ and that the state change is a rare event when compared to the shock in state H. Formally,

Assumption $1 \theta^{H}-\theta^{L}>p^{H}+p^{L}$.

By observing whether the shock occurs or not she continuously revises her subjective shock probability in a Bayesian manner. Let $\theta_{t}$ denote the true shock probability at time $t$, which is unknown to her. Using information available up to time $t$, she forms a belief that $\theta_{t}=\theta^{H}$ with probability $f_{t}\left(\theta^{H}\right)$ and $\theta_{t}=\theta^{L}$ with probability $f_{t}\left(\theta^{L}\right)$. Obviously,

$$
f_{t}\left(\theta^{L}\right)+f_{t}\left(\theta^{H}\right)=1 \quad \text { for any } t \text {. }
$$

In order to find how she updates $f_{t}\left(\theta^{i}\right)$ from $t$ to $t+\Delta t,{ }^{4}$ we first obtain the subjective probability that the shock does not occur between $t$ and $t+\Delta t$ for given $f_{t}\left(\theta^{i}\right)$. It is denoted by $F_{t}\left[S_{(t, t+\Delta t]}=\phi\right]$, where $F_{t}[\cdot]$ is a probability operator based on information available at $t, S_{(a, b]}$ is the set of dates on which the shock actually occurs during $(a, b]$, and $\phi$ the empty set. Since the underlying state is either $\mathrm{H}$ or $\mathrm{L}$ at time $t+\Delta t$, this probability is divided into two components, $F_{t}\left[S_{(t, t+\Delta t]}=\phi \cap \theta_{t+\Delta t}=\theta^{H}\right]$ and $F_{t}\left[S_{(t, t+\Delta t]}=\phi \cap \theta_{t+\Delta t}=\theta^{L}\right]$.

\footnotetext{
${ }^{4}$ Time interval $\Delta t$ is taken to be so short that the probability that the liquidity shock and a state change coexist in the interval is negligible.
} 
Each of the two components is further divided into two probabilities. The former is the sum of the probability that 'the state is $\mathrm{H}$ at time $t$ and neither the state change nor the shock occurs during the interval' and the probability that 'the present state is $\mathrm{L}$ and the state changes to $\mathrm{H}$ during the interval.' It is ${ }^{5}$

$$
F_{t}\left[S_{(t, t+\Delta t]}=\phi \cap \theta_{t+\Delta t}=\theta^{H}\right]=\left(1-\left(\theta^{H}+p^{H}\right) \Delta t\right) f_{t}\left(\theta^{H}\right)+p^{L} \Delta t f_{t}\left(\theta^{L}\right)
$$

Similarly, the latter is

$$
F_{t}\left[S_{(t, t+\Delta t]}=\phi \cap \theta_{t+\Delta t}=\theta^{L}\right]=\left(1-\left(\theta^{L}+p^{L}\right) \Delta t\right) f_{t}\left(\theta^{L}\right)+p^{H} \Delta t f_{t}\left(\theta^{H}\right)
$$

Summing up (2) and (3) yields

$$
F_{t}\left[S_{(t, t+\Delta t]}=\phi\right]=1-\theta_{t}^{e} \Delta t
$$

where $\theta_{t}^{e}$ represents the expected (or subjective) probability of the shock per unit time at time $t$ :

$$
\theta_{t}^{e} \equiv \theta^{H} f_{t}\left(\theta^{H}\right)+\theta^{L} f_{t}\left(\theta^{L}\right)
$$

Let us consider how the representative household updates her belief if she eventually finds that the shock did not occur during $(t, t+\Delta t]$. In this case the information that $S_{(t, t+\Delta t]}=\phi$ is added to her knowledge. Thus, using Bayes' law we find updated subjective probability $f_{t+\Delta t}\left(\theta^{i}\right)$ to be

$$
\begin{aligned}
f_{t+\Delta t}\left(\theta^{i}\right) & \equiv F_{t+\Delta t}\left[\theta_{t+\Delta t}=\theta^{i}\right]=F_{t}\left[\theta_{t+\Delta t}=\theta^{i} \mid S_{(t, t+\Delta t]}=\phi\right] \\
& =\frac{F_{t}\left[S_{(t, t+\Delta t]}=\phi \cap \theta_{t+\Delta t}=\theta^{i}\right]}{F_{t}\left[S_{(t, t+\Delta t]}=\phi\right]} .
\end{aligned}
$$

Since the numerator is given by (2) or (3) and the denominator by $(4), f_{t+\Delta t}\left(\theta^{H}\right)$ equals $^{6}$

$$
f_{t+\Delta t}\left(\theta^{H}\right)=\frac{\left(1-\left(\theta^{H}+p^{H}\right) \Delta t\right) f_{t}\left(\theta^{H}\right)+p^{L} \Delta t f_{t}\left(\theta^{L}\right)}{1-\theta_{t}^{e} \Delta t} .
$$

\footnotetext{
${ }^{5}$ Throughout the paper we ignore the second-order term of $\Delta t$ and higher because $\Delta t \rightarrow 0$.

${ }^{6} f_{t+\Delta t}\left(\theta^{L}\right)$ is analogously obtained. From (1) it equals $1-f_{t+\Delta t}\left(\theta^{H}\right)$.
} 
From this equation we derive the time derivative of $f_{t}\left(\theta^{H}\right)$ :

$$
\begin{aligned}
\frac{d f_{t}\left(\theta^{H}\right)}{d t} & =\lim _{\Delta t \rightarrow 0} \frac{f_{t+\Delta t}\left(\theta^{H}\right)-f_{t}\left(\theta^{H}\right)}{\Delta t} \\
& =\left(\theta_{t}^{e}-\theta^{H}-p^{H}\right) f_{t}\left(\theta^{H}\right)+p^{L} f_{t}\left(\theta^{L}\right) .
\end{aligned}
$$

We next consider the case where the shock occurs during $(t, t+\Delta t]$. Since

$$
F_{t}\left[S_{(t, t+\Delta t]} \neq \phi \cap \theta_{t+\Delta t}=\theta^{i}\right]=\theta^{i} f_{t}\left(\theta^{i}\right) \Delta t \quad \text { for } \quad i \in\{L, H\}
$$

the probability that the shock occurs is

$$
F_{t}\left[S_{(t, t+\Delta t]} \neq \phi\right]=\left(\theta^{H} f_{t}\left(\theta^{H}\right)+\theta^{L} f_{t}\left(\theta^{L}\right)\right) \Delta t=\theta_{t}^{e} \Delta t
$$

which is consistent with (4). From Bayes' law dividing (7) by (8) gives the updated subjective probability that $\theta_{t+\Delta t}=\theta^{i}$ under the condition that the shock occurs during $(t, t+\Delta t]$. It is

$$
f_{t}\left(\theta^{i}\right)=\lim _{t^{\prime} \rightarrow t-0} \frac{\theta^{i} f_{t^{\prime}}\left(\theta^{i}\right)}{\theta_{t^{\prime}}^{e}} \equiv \frac{\theta^{i} f_{t-0}\left(\theta^{i}\right)}{\theta_{t-0}^{e}},
$$

where subscript $t-0$ represents the state just before $t$.

Finally, we obtain the dynamics of subjective probability $\theta_{t}^{e}$. From (1) and (5),

$$
f_{t}\left(\theta^{H}\right)=\frac{\theta_{t}^{e}-\theta^{L}}{\theta^{H}-\theta^{L}}, \quad f_{t}\left(\theta^{L}\right)=\frac{\theta^{H}-\theta_{t}^{e}}{\theta^{H}-\theta^{L}}
$$

Substituting (6) and (10) into the time derivative of (5) yields the time derivative of $\theta_{t}^{e}$ in the case where the shock does not occur at time $t$ :

$$
\dot{\theta}_{t}^{e}=\left(\theta_{t}^{e}-\theta^{L}-p^{L}\right)\left(\theta_{t}^{e}-\theta^{H}-p^{H}\right)-p^{L} p^{H} \equiv g\left(\theta_{t}^{e}\right) \quad \text { for } t \notin S_{(0, \infty)} .
$$

Under Assumption 1, this function has an 'U'-shape as illustrated in figure 1. The figure shows that

$$
\begin{aligned}
g(\theta) & \lesseqgtr 0 \Longleftrightarrow \theta \gtreqless \theta^{*} \text { for any } \theta \in\left[\theta^{L}, \theta^{H}\right], \text { where } \\
\theta^{*} & \equiv \frac{\theta^{L}+\theta^{H}+p^{L}+p^{H}-\sqrt{\left(\theta^{H}+p^{H}-\theta^{L}-p^{L}\right)^{2}+4 p^{L} p^{H}}}{2} \in\left(\theta^{L}, \theta^{H}\right) .
\end{aligned}
$$


Similarly, by substituting (9) and (10) into (5) we obtain the value of $\theta_{t}^{e}$ as a function of $\theta_{t-0}^{e}$ in the case where the shock does occur at time $t$.

$$
\theta_{t}^{e}=\theta^{L}+\theta^{H}-\frac{\theta^{L} \theta^{H}}{\theta_{t-0}^{e}} \equiv h\left(\theta_{t-0}^{e}\right) \quad \text { for } t \in S_{(0, \infty)} .
$$

As shown in Figure 2, $h(\theta)$ satisfies

$$
h\left(\theta^{H}\right)=\theta^{H} \text {, and } \theta^{e}<h\left(\theta^{e}\right)<\theta^{H} \text { for all } \theta^{e} \in\left(\theta^{L}, \theta^{H}\right) .
$$

Equations (11) and (13) describe the dynamics of $\theta_{t}^{e}$ with and without the shock respectively. It continuously declines to steady state value $\theta^{*}$ as long as the shock does not occur, but discretely jumps upward when it occurs. ${ }^{7}$ Intuitively, in the absence of the shock people gradually become more and more optimistic and confident that the economy is in state L. Thus, their subjective probability of the shock gradually declines and converges to $\theta^{*}{ }^{8}$ Due to the U-shape of function $g\left(\theta_{t}^{e}\right)$, the speed of adjusting their belief is slower when $\theta_{t}^{e}$ is near either $\theta^{*}$ or $\theta^{H}$ than when it is in the middle.

Conversely, when the shock is observed, people discretely change their expectation about the present state. Since $h\left(\theta^{e}\right)$ is located above the 45 -degree line in Figure 2, the more often people observe the shock, the more strongly people believe that they are in state $\mathrm{H}$, and hence $\theta_{t}^{e}$ becomes closer to $\theta^{H}$. In this way $\theta_{t}^{e}$ fluctuates between $\theta^{*}$ and $\theta^{H}$.

${ }^{7}$ From (11) and (13), we find that $\theta_{t}^{e}$ is trapped within interval $\left(\theta^{*}, \theta^{H}\right]$ in the long run. Since we are interested in the long-term behavior of the economy, it is assumed throughout this paper that $\theta_{t}^{e}$ is always within $\left(\theta^{*}, \theta^{H}\right]$. Under this assumption, $\theta_{t}^{e}$ always declines while the shock does not occur.

${ }^{8} \theta_{t}^{e}$ never becomes lower than $\theta^{*}\left(>\theta^{L}\right)$ since people take into account the possibility that state $\mathrm{L}$ might have changed to state $\mathrm{H}$ even though the shock does not occur. 


\section{Liquidity Preference and Consumption Behav- ior}

In this section, we investigate how the aforementioned fluctuations in the representative household's belief affects her liquidity preference and consumption behavior. Before doing that, we briefly describe the basic structure and production side of the economy.

The economy is inhabited by the representative households with measure one. Each household is infinitely lived and supplies labor to a representative firm. The firm inputs only labor to produce the commodity according to $y_{t}=\bar{y} x_{t}$, where $y_{t}$ and $x_{t}$ are respectively the level of output and that of labor input. ${ }^{9}$ Input-output coefficient $\bar{y}$ is assumed to be constant. Since there is no investment in our setting and thus consumption $c_{t}$ equals total commodity demand, total labor demand $x_{t}$ is

$$
x_{t}=c_{t} / \bar{y}
$$

In this economy, money affects real variables through the sluggishness of nominal wage adjustment. Instead of explicitly introducing the adjustment cost of nominal wages, we simply assume that there is a reduced-form relationship between labor demand $x_{t}$ and the rate of nominal wage adjustment $\dot{W}_{t} / W_{t}$,

$$
\dot{W}_{t} / W_{t}=\omega\left(x_{t}-1\right), \quad \omega^{\prime}(\cdot) \geq 0, \omega(0)=0
$$

i.e., the rate of nominal wage adjustment is an increasing function of labor demand in excess of the 'natural' level, the latter being normalized to unity. Given $W_{t}$, the perfect adjustment of commodity price $P_{t}$ always yields

$$
W_{t} / P_{t} \equiv w_{t}=\bar{y}
$$

\footnotetext{
${ }^{9}$ Labor supplied by each household is potentially differentiated. In that case ' $y_{t}=\bar{y} x_{t}$ ' should be considered as the outcome when the representative firm employs the same amount of labor from each household.
} 
which shows that real wage $w_{t}$ is constant. Thus, from (15), the inflation rate is determined as a function of commodity demand $c_{t}$ :

$$
\pi_{t} \equiv \dot{P}_{t} / P_{t}=\omega\left(c_{t} / \bar{y}-1\right)
$$

Next, we describe the demand side. We extend the money-in-the-utility-function framework to include the random liquidity shock. The representative household gains utility only from consumption $c_{t}$ when the shock does not occur. However, when it occurs, she gains utility not only from consumption but also from money holding $m_{t}$. Her expected utility $E U_{t}$ is therefore given by

$$
E U_{t}=E_{t}\left[\int_{t}^{\infty} u\left(c_{\tau}\right) e^{-\rho(\tau-t)} d \tau+\sum_{\tau \in S_{(t, \infty)}} \beta m_{\tau} e^{-\rho(\tau-t)}\right]
$$

where $\rho$ is her subjective discount rate, $\beta$ is a constant specifying the marginal utility of money holding, and $S_{(t, \infty)}$ is the set of future dates on which the shock occurs. Instantaneous felicity from consumption $u(\cdot)$ is twice differentiable and satisfies the Inada conditions.

The household chooses assets among money and contingent claims for future commodities. However, since all households are identical, money is the only asset that they hold after all arbitrage opportunities are exploited. ${ }^{10}$ We assume that nominal money supply is constant and that there is no tax-cum-subsidy. ${ }^{11}$ Thus, the flow budget equation is

$$
\dot{m}_{t}=w_{t} x_{t}-\pi_{t} m_{t}-c_{t}
$$

Having the belief mentioned in the previous section, the representative household chooses the time paths of consumption and money holding so as to maximize the expected utility (18) subject to (19).

\footnotetext{
${ }^{10}$ Since the profits of firms are always zero in the present setting, the ownership of firms has no value.

${ }^{11}$ The assumption of constant money supply is not essential. In fact, as shown below, the level of money stock does not affect the household behavior in the present setting.
} 
Objective function (18) depends on only $c_{t}, m_{t}$ and the expected pattern of the shock, the last of which is fully described by $\theta_{t}^{e}$ since $\theta_{t}^{e}$ is a sufficient statistic for $\theta_{t}$ that governs the current and future probabilities of the shock. Constraint (19) depends on $w_{t}, x_{t}$ and $\pi_{t}$, all of which are determined by only $c_{t}$ on the equilibrium path, as seen from (14), (16) and (17). Therefore, from the perspective of the household that determines $c_{t}$, the current status is fully summarized by $m_{t}$ and $\theta_{t}^{e}$. Thus, given the recursive structure of the model, the movement of $c_{t}$ on the path of stationary dynamics must completely be expressed as a function of $m_{t}$ and $\theta_{t}^{e}{ }^{12}$ Furthermore, since objective function (18) and constraint (19) are both linear in $m_{t}$, the optimal choice of $c_{t}$ is independent of the level of $m_{t} \cdot{ }^{13}$ Thus, $c_{t}$ should be a function of only $\theta_{t}^{e}$ :

$$
c_{t}=C\left(\theta_{t}^{e}\right) \text { for all } t \text {. }
$$

Since $\theta_{t}^{e}$ fluctuates within interval $\left(\theta^{*}, \theta^{H}\right]$, as shown in the previous section, we only need to characterize the shape of function $C\left(\theta_{t}^{e}\right)$ in this interval. ${ }^{14}$ To this end we examine the first-order conditions for the household's optimizing behavior.

Let $1-\mu\left(\theta_{t}^{e}\right) \Delta t$ denote the price of the claim to a unit of the commodity at $t+\Delta t$ measured in terms of the commodity at $t$ under the condition that the shock does not occur between $t$ and $t+\Delta t$. Note that it is a function of $\theta_{t}^{e}$ because the value

\footnotetext{
${ }^{12}$ This strategy for finding stationary dynamics is analogous to Lucas (1978) who analyses the determination of equilibrium price behavior under an exogenous production shock that follows a Markov process.

${ }^{13}$ The linearity of the utility function with respect to $m_{t}$ is assumed primarily for showing how the fluctuation in liquidity preference affects the consumption path of the utility-maximizing household in the simplest setting. When the marginal utility of holding money is variable, we actually find that the equilibrium dynamics of $c_{t}$ depends on both $\theta_{t}^{e}$ and $m_{t}$. It substantially complicates the analysis but does not affect our main results, such as the pattern of recovery.

${ }^{14}$ In the following, it is assumed that $\theta_{t}^{e} \in\left(\theta^{*}, \theta^{H}\right]$ unless otherwise noticed.
} 
of the claim depends on the probability with which the contingent event occurs. ${ }^{15}$ If the shock does not occur during the interval, consumption increases from $C\left(\theta_{t}^{e}\right)$ to $C\left(\theta_{t}^{e}+g\left(\theta_{t}^{e}\right) \Delta t\right)$ since $\theta_{t}^{e}$ changes by the amount of $g\left(\theta_{t}^{e}\right) \Delta t$, as shown by (11). Since the probability that the shock does not occur during this interval is $1-\theta_{t}^{e} \Delta t$, as given by (4), the first-order condition between the present and future consumption under the condition that the shock does not occur is

$$
\left(1-\mu\left(\theta_{t}^{e}\right) \Delta t\right) u^{\prime}\left(C\left(\theta_{t}^{e}\right)\right)=\left(1-\theta_{t}^{e} \Delta t\right) u^{\prime}\left(C\left(\theta_{t}^{e}+g\left(\theta_{t}^{e}\right) \Delta t\right)\right) e^{-\rho \Delta t} .
$$

From this equation in which $\Delta t \rightarrow 0$ we derive

$$
\mu\left(\theta_{t}^{e}\right)=\rho+\theta_{t}^{e}+\frac{C^{\prime}\left(\theta_{t}^{e}\right)}{C\left(\theta_{t}^{e}\right)} \gamma\left(C\left(\theta_{t}^{e}\right)\right) g\left(\theta_{t}^{e}\right) \text { for all } \theta_{t}^{e}
$$

where $\gamma(c) \equiv-u^{\prime \prime}(c) c / u^{\prime}(c)$.

Analogously, let $\nu\left(\theta_{t}^{e}\right) \Delta t$ denote the price of the contingent claim to a unit of the commodity at $t+\Delta t$ under the condition that the shock does occur between $t$ and $t+\Delta t$. When the shock occurs, consumption jumps from $C\left(\theta_{t}^{e}\right)$ to $C\left(h\left(\theta_{t}^{e}\right)\right)$, as seen from (13). Since the shock probability is $\theta_{t}^{e} \Delta t$, as shown by (8), the first-order condition between the present and the future consumption in this case is

$$
\nu\left(\theta_{t}^{e}\right) \Delta t \cdot u^{\prime}\left(C\left(\theta_{t}^{e}\right)\right)=\theta_{t}^{e} \Delta t \cdot u^{\prime}\left(C\left(h\left(\theta_{t}^{e}\right)\right)\right) e^{-\rho \Delta t} .
$$

By making $\Delta t \rightarrow 0$ in this equation we find

$$
\nu\left(\theta_{t}^{e}\right)=\frac{\theta_{t}^{e} u^{\prime}\left(C\left(h\left(\theta_{t}^{e}\right)\right)\right)}{u^{\prime}\left(C\left(\theta_{t}^{e}\right)\right)} \text { for all } \theta_{t}^{e}
$$

Next, let us consider the arbitrage between these contingent claims and a risk-free asset. Let $r_{t}$ be the real interest rate of a risk-free asset, then the price of a risk-free claim to the future commodity at $t+\Delta t$ is $e^{-r_{t} \Delta t}$. Since the claim is equivalent to the asset of the synthesis of the claim contingent on the absence of the shock whose

\footnotetext{
${ }^{15}$ Note also that consumption (which affects the marginal utility of consumption) depends on $\theta_{t}^{e}$.
} 
price is $1-\mu\left(\theta_{t}^{e}\right) \Delta t$ and that conditional on its presence whose price is $\nu\left(\theta_{t}^{e}\right) \Delta t$, the no-arbitrage condition requires

$$
e^{-r_{t} \Delta t}=1-\mu\left(\theta_{t}^{e}\right) \Delta t+\nu\left(\theta_{t}^{e}\right) \Delta t
$$

As $\Delta t \rightarrow 0$, it reduces to

$$
r_{t}=\mu\left(\theta_{t}^{e}\right)-\nu\left(\theta_{t}^{e}\right) \text { for all } t
$$

Substituting (21) and (22) into (23) yields

$$
C^{\prime}\left(\theta_{t}^{e}\right)=\frac{C\left(\theta_{t}^{e}\right)}{\gamma\left(C\left(\theta_{t}^{e}\right)\right) g\left(\theta_{t}^{e}\right)}\left[r_{t}-\rho+\theta_{t}^{e}\left(\frac{u^{\prime}\left(C\left(h\left(\theta_{t}^{e}\right)\right)\right)}{u^{\prime}\left(C\left(\theta_{t}^{e}\right)\right)}-1\right)\right] .
$$

Applying (11) and (20) to (24) leads to the dynamics of $c_{t}\left(\equiv C\left(\theta_{t}^{e}\right)\right)$ while the shock does not occur:

$$
\frac{\dot{c}_{t}}{c_{t}}=\frac{1}{\gamma\left(c_{t}\right)}\left[r_{t}-\rho+\theta_{t}^{e}\left(\frac{u^{\prime}\left(C\left(h\left(\theta_{t}^{e}\right)\right)\right)}{u^{\prime}\left(c_{t}\right)}-1\right)\right] \quad \text { for } t \notin S_{(0, \infty)},
$$

which is the Keynes-Ramsey rule in the present setting. Note that it is the same as the standard one except for the third term in brackets of the right-hand side. This term represents a jump in the marginal utility caused by the shock. If $c_{t}$ declines after the shock, causing the marginal utility of consumption to increase, this term is positive and thus the growth rate of $c_{t}$ is higher than would obtain in the standard Ramsey model. That is, since the representative household anticipates a possible increase in the marginal utility of consumption, she tries to reallocate consumption from the present to the future, raising the growth rate of consumption during the period without the shock.

Having examined the household's intertemporal optimization of consumption, we now turn to the optimal choice between consumption and money holding. By holding a one-unit money between time $t$ and $t+\Delta t$, the household loses $\left(r_{t}+\pi_{t}\right) \Delta t$ units of consumption, or equivalently $\left(r_{t}+\pi_{t}\right) u^{\prime}\left(c_{t}\right) \Delta t$ units in terms of utility, when compared to holding a unit of risk-free asset during this period. At this cost a one-unit increase 
in real money holding raises the household's utility (18) by $\beta$ if the shock occurs. Since the subjective probability that the shock occurs between $t$ and $t+\Delta t$ is $\theta_{t}^{e} \Delta t$, the increase in the expected utility is $\beta \theta_{t}^{e} \Delta t$. The marginal benefit should equal the marginal cost on the optimal path, which yields the first-order condition between money holding and consumption:

$$
r_{t}+\pi_{t}=\frac{\beta \theta_{t}^{e}}{u^{\prime}\left(c_{t}\right)} \text { for all } t
$$

Equation (26) implies the well-known property-i.e., the marginal rate of substitution between consumption and real money holding equals the nominal rate of interest. Substituting (17) into (26) and rearranging terms, the real interest rate is written as a function of $\theta_{t}^{e}$ and $c_{t}$ :

$$
\begin{aligned}
& r_{t}=\frac{\beta \theta_{t}^{e}}{u^{\prime}\left(c_{t}\right)}-\omega\left(\frac{c_{t}}{\bar{y}}-1\right) \equiv R\left(\theta_{t}^{e}, c_{t}\right), \quad \text { satisfying } \\
& R_{\theta}\left(\theta^{e}, c\right) \equiv \partial R\left(\theta^{e}, c\right) / \partial \theta^{e}=\frac{\beta}{u^{\prime}\left(c_{t}\right)}>0 \quad \text { for all } \theta_{t}^{e}>0 \text { and } c_{t}>0 .
\end{aligned}
$$

From (24) and (27), the optimizing household behavior is summarized as

$$
\begin{aligned}
& C^{\prime}\left(\theta_{t}^{e}\right)=\frac{C\left(\theta_{t}^{e}\right)}{\gamma\left(C\left(\theta_{t}^{e}\right)\right) g\left(\theta_{t}^{e}\right)}\left[R\left(\theta_{t}^{e}, C\left(\theta_{t}^{e}\right)\right)-\rho+\theta_{t}^{e}\left(\frac{u^{\prime}\left(C\left(h\left(\theta_{t}^{e}\right)\right)\right)}{u^{\prime}\left(C\left(\theta_{t}^{e}\right)\right)}-1\right)\right] \\
& \quad \text { for all } \theta_{t}^{e} \in\left(\theta^{*}, \theta^{H}\right] .
\end{aligned}
$$

Function $C(\cdot)$ is determined so that it satisfies differential equation (28). To pin down $C(\cdot)$, however, we also need a boundary condition. If $\dot{c}_{t} / c_{t}$ remains positive as $\theta_{t}^{e}$ approaches steady-state value $\theta^{*}$, then $c_{t}$ unboundedly explodes. Conversely, if $\dot{c}_{t} / c_{t}$ remains negative as $\theta_{t}^{e} \rightarrow \theta^{*}, c_{t}$ converges to zero, violating the transversality condition. We rule out such paths by imposing a boundary condition: ${ }^{16}$

$$
\lim _{\theta^{e} \rightarrow \theta^{*}} R\left(\theta^{e}, C\left(\theta^{e}\right)\right)-\rho+\theta^{e}\left(\frac{u^{\prime}\left(C\left(h\left(\theta^{e}\right)\right)\right)}{u^{\prime}\left(C\left(\theta^{e}\right)\right)}-1\right)=0,
$$

under which $\dot{c}_{t} / c_{t}$ given by (25) approaches zero as $\theta_{t}^{e} \rightarrow \theta^{*}$.

\footnotetext{
${ }^{16}$ Throughout this paper, we use operator 'lim' to denote the right-hand limit.
} 
Equations (28) and (29) determine the whole shape of $C\left(\theta_{t}^{e}\right)$ within interval $\left(\theta^{*}, \theta^{H}\right]$. Once it is determined, applying the dynamics of $\theta_{t}^{e}$ given by (11) and (13) to it provides the dynamic path of $c_{t}$.

\section{Business Cycle}

This section investigates the shape of the consumption path determined by (28) and (29). Note that the right-hand side of differential equation (28) includes $C(h(\theta))$ along with $\theta$ and $C(\theta)$, implying that we cannot simply illustrate a phase diagram. ${ }^{17}$ Therefore, we theoretically examine basic properties of function $C(\theta)$ and then numerically obtain a typical shape of it.

For the tractability of the analysis we assume the following two properties regarding $u(\cdot)$ and $\omega(\cdot)$, which are stated in terms of $R(\cdot)$ defined by $(27)$.

Assumption $2 \lim _{c \rightarrow 0} R\left(\theta^{H}, c\right)<\rho$ and $\lim _{c \rightarrow \infty} R\left(\theta^{*}, c\right)>\rho$.

Assumption $3 R_{c}\left(\theta^{e}, c\right)$ is continuous and positive for all $\theta^{e} \in\left[\theta^{*}, \theta^{H}\right]$ and $c>0$, where $R_{c}\left(\theta^{e}, c\right) \equiv \partial R\left(\theta^{e}, c\right) / \partial c$.

Intuitively, function $R(\cdot)$ represents the household's preference for liquidity-i.e., the expected utility gain minus capital loss from holding money for a unit time, as shown by (27). If it is stronger than her preference for present consumption $\rho$, she postpones consumption and holds more money. Therefore, in Assumption 2 the former property implies that she prefers consumption to liquidity holding when her consumption is quite low even if she expects the highest shock probability $\theta^{H}$. The latter property implies that she prefers liquidity holding to consumption when her consumption is sufficiently high even if she expects the lowest shock probability

\footnotetext{
${ }^{17}$ In mathematical term, this type of equation is called a difference-differential equation or a delay difference equation.
} 
$\theta^{*}$. Assumption 3 extends this relationship between $c_{t}$ and $R(\cdot)$ to a smooth and monotonic one under a given $\theta_{t}^{e}$-i.e., as consumption increases, liquidity preference rises as long as the state of expectation about the shock is unchanged.

Under the two assumptions we find the existence, and a few properties, of function $C\left(\theta^{e}\right)$ that satisfies $(28)$ and $(29)$ :

Proposition 1 Under Assumptions 2 and 3, there exists a unique function $C\left(\theta^{e}\right)$ that satisfies (28) and (29). It is strictly downward sloping for any $\theta^{e} \in\left(\theta^{*}, \theta^{H}\right]$ and has positive and finite upper and lower bounds $\bar{c}$ and $\underline{c}$ that are given by

$$
R\left(\theta^{*}, \bar{c}\right)=\rho, \quad R\left(\theta^{H}, \underline{c}\right)=\rho .
$$

Proof. See Appendix A.

Given initial belief $\theta_{0}^{e}$ and the history of the liquidity shock $S_{(0, t]}$, the path of $\theta_{t}^{e}$ is uniquely determined by (11) and (13). Therefore, the uniqueness of function $C(\cdot)$ implies that of the consumption path: $c_{t}=C\left(\theta_{t}^{e}\right)$. The negative relationship between $\theta_{t}^{e}$ and $c_{t}$ provides an intuitive figure of the dynamics. Figure 1 shows $\dot{\theta}_{t}^{e}<0$ for any $\left(\theta^{*}, \theta^{H}\right]$, implying that subjective shock probability $\theta_{t}^{e}$ gradually declines while the shock does not occur. Thus, preference for liquidity gradually decreases and consumption grows. When the shock occurs, people discretely increase $\theta_{t}^{e}$ to $h\left(\theta_{t}^{e}\right)$, as illustrated in Figure 2, causing a negative jump in consumption to occur. If the shock does not occur for a while, people again gradually become optimistic and raise consumption. In this way, consumption persistently fluctuates within finite interval $[\underline{c}, \bar{c}]$.

To obtain a typical shape of function $C\left(\theta^{e}\right)$ and the dynamics of consumption more clearly, we numerically analyze the dynamics by assuming

$$
u\left(c_{t}\right)=\log c_{t} \text { and } \omega\left(x_{t}-1\right)=\alpha \cdot\left(x_{t}-1\right) \text { where } \alpha \text { is constant. }
$$


In this setting, Assumptions 2 and 3 reduce to the following: ${ }^{18}$

$$
\beta \bar{y} \theta^{*}>\alpha, \quad \rho>\alpha
$$

In the numerical calculation we choose parameter values so that these conditions as well as Assumption 1 are satisfied. ${ }^{19}$

Figure 3 illustrates the shape of $C\left(\theta_{t}^{e}\right)$ obtained from the numerical analysis. It is in fact downward sloping from $c^{*} \equiv C\left(\theta^{*}\right)$ to $c^{H} \equiv C\left(\theta^{H}\right)$. If the shock does not occur while $\theta_{t}^{e}$ moves from $\theta^{H}$ to $\theta^{*}$ according to equation (11), $C\left(\theta_{t}^{e}\right)$ moves along the solid curve in Figure 4. Note that consumption first grows slowly, gradually accelerates, and eventually slows down again as it approaches $c^{*}$-i.e., it traces an S-shaped trajectory.

The intuition behind is clear from the U-shape of function $g\left(\theta_{t}^{e}\right)$ as depicted in Figure 1. If people strongly believe that they are in state $\mathrm{H}$ and thus $\theta_{t}^{e}$ is very close to $\theta^{H}$, they do not significantly alter their pessimistic view for a while. In fact, the speed of change in $\theta_{t}^{e}$, given by $\left|g\left(\theta_{t}^{e}\right)\right|$, is then small and thus consumption increases very slowly. As the period without the shock lasts, $\theta_{t}^{e}$ decreases and $\left|g\left(\theta_{t}^{e}\right)\right|$ increases, as Figure 1 shows. People become more and more optimistic and thus $\theta_{t}^{e}$ declines faster, which accelerates the recovery speed of consumption. As $\theta_{t}^{e}$ approaches to steady-state value $\theta^{*}$, people become quite confident that they are in state $\mathrm{L}$, and hence an additional period without the shock provides little information. $\left|g\left(\theta_{t}^{e}\right)\right|$ approaches zero and the growth rate of consumption converges to zero.

\footnotetext{
${ }^{18}$ Using a money-in-utility model without uncertainty Ono (1994, pp.86-88; 2001) shows that in the case where $\beta \bar{y}>\alpha$ and $\rho>\alpha$ there is a unique saddle-stable path. Furthermore, the path accommodates a persistent demand shortage when $\rho<\beta \bar{y}$, whereas it reaches a full-employment steady state when $\rho>\beta \bar{y}$. The present condition is the same as his condition except that the former includes shock probability $\theta^{*}$.

${ }^{19}$ Specifically, $\theta^{H}=.4, \theta^{L}=.05, p^{H}=.025, p^{L}=.1, \bar{y}=1, \rho=.05, \alpha=.025$, and $\beta=.4$. Under these parameter values, we obtain $\theta^{*} \approx .069, c^{*} \approx 1.3$ and $c^{H} \approx .60$. The details of the numerical procedure are described in Appendix B.
} 
Once the liquidity shock occurs, however, their consumption jumps downward since the subjective shock probability jumps upward, raising their liquidity preference. Consumption falls from $C\left(\theta_{t}^{e}\right)$ to $C\left(h\left(\theta_{t}^{e}\right)\right)$, the latter being depicted by the dashed curve in Figure 4 (where vertical arrows express the magnitude of each fall). Thereafter the recovery process 'restarts' from the point that corresponds to the decreased level of consumption (as indicated by horizontal arrows) and consumption again traces the solid curve. If the shock continually occurs for a short period, the subjective probability successively increases and $C\left(\theta_{t}^{e}\right)$ approaches the lowest level $c^{H}$. Thereafter, consumption recovers along the S-shaped trajectory, as mentioned above.

Finally, by simulating the Markov process of the underlying state and the Poisson process for the shock, we numerically obtain an example of the realized time paths of $\theta_{t}, \theta_{t}^{e}$ and $c_{t}$. Figure 5 illustrates them. Consumption in fact traces an S-shaped path especially after it vastly declines. Since the inflation rate is given by (17) and $\bar{y}$ is located between $c^{*}$ and $c^{H}$ under the present parameter values, in the recovery process serious deflation initially occurs, then its rate reduces, and eventually a boom comes and inflation arises after $c$ exceeds $\bar{y}$. If the shock does not frequently occur and hence consumption does not seriously decline, the recovery process starts before deflation occurs.

It is also worth noting that realized booms and depressions do not exactly match the underlying state of the economy but follow the subjective probability that people have in mind. Even when the economy switches to state $\mathrm{H}$ and thus the true probability of the shock jumps up, people do not increase money holding until they actually observe it. Analogously, even if the true shock probability jumps down, they still keep strong liquidity preference and thus the recovery speed is very slow for a while once they become very pessimistic. 


\section{Conclusions}

Liquidity preference depends on people's belief about how frequently they encounter crises in which liquidity is needed. This paper has examined the way they update the belief based on Bayesian inference and its effect on their preference for liquidity holding over consumption, in a circumstance where the economy shifts between two unobservable states with different probabilities of the liquidity shock. Each time they observe the shock, they raise their subjective probability of being in the more dangerous state and increase preference for holding money over consumption. The longer the period without the shock lasts, the larger probability people attach to the safer state and increase preference for consumption over money holding. With incomplete nominal wage adjustment, such movements in liquidity preference generate demand-driven business cycles.

The magnitude and persistence of fluctuations in consumption depend on the realized frequency of the shock, which does not necessarily match the underlying state of the economy. As long as the shock occurs sparsely in time, it has only a minor effect on the belief and economic recovery after it is fast. However, if people observe the shock many times for a short while, they hold a strong belief of being in the more dangerous state and reduce consumption a lot. Once it occurs, it takes a long time for them to reverse their belief and increase consumption. In this process, the recovery speed is first slow, then gradually accelerates, and eventually declines, tracing an S-shaped curve. 


\section{Appendix A: Proof of Proposition 1}

\section{Proof of $C(\theta)$ to be downward sloping ${ }^{20}$}

Before starting the proof we define $D(\theta)$ :

$$
D(\theta) \equiv R(\theta, C(\theta))-\rho+\theta\left(\frac{u^{\prime}(C(h(\theta)))}{u^{\prime}(C(\theta))}-1\right) .
$$

Since $D(\theta)$ is the expression in brackets of (28) and (11) shows $g(\theta)$ to be negative for all $\theta \in\left(\theta^{*}, \theta^{H}\right]$,

$$
C^{\prime}(\theta) \lesseqgtr 0 \Longleftrightarrow D(\theta) \gtreqless 0
$$

Using function $D(\theta)$ we first prove

Lemma 1 Suppose that $C(\theta)$ satisfies (28) and that there exists $\theta^{0} \in\left[\theta^{*}, \theta^{H}\right)$ satisfying $\lim _{\theta \rightarrow \theta^{0}} D(\theta)=0$. Then, under Assumption 3, $C(\theta)$ is strictly downward sloping for all $\theta \in\left(\theta^{0}, \theta^{H}\right]$.

Proof: If Lemma 1 does not hold and hence $C(\cdot)$ is weakly upward sloping somewhere in $\left(\theta^{0}, \theta^{H}\right]$, either of the following must be the case.

(i) There exists some $\theta^{A} \in\left[\theta^{0}, \theta^{H}\right)$ such that $\lim _{\theta \rightarrow \theta^{A}} D(\theta)=0$ and $C^{\prime}(\theta) \geq 0$ for all $\theta \in\left(\theta^{A}, \theta^{H}\right]$.

(ii) There exist some $\theta^{A} \in\left[\theta^{0}, \theta^{H}\right)$ and $\theta^{B} \in\left(\theta^{A}, \theta^{H}\right)$ such that $\lim _{\theta \rightarrow \theta^{A}} D(\theta)=$ $D\left(\theta^{B}\right)=0, C^{\prime}(\theta) \geq 0$ for all $\theta \in\left(\theta^{A}, \theta^{B}\right]$, and $C^{\prime}(\theta) \leq 0$ for all $\theta \in\left(\theta^{B}, \theta^{H}\right]$.

Intuitively, if the lemma is false, we can choose interval $\left(\theta^{A}, \theta^{B}\right]$ in which function $C(\theta)$ is weakly increasing, $\theta^{A}$ is either a local minimum or $\theta^{0}$, and $\theta^{B}$ is either a local maximum or $\theta^{H}$. If there are multiple intervals of such, we choose the rightmost one. We shall find neither (i) nor (ii) to be valid.

\footnotetext{
${ }^{20}$ In Appendices $\mathrm{A}$ and $\mathrm{B}$ we use $\theta$ instead of $\theta_{t}^{e}$ to minimize notation.
} 
We first show that case (i) leads to a contradiction. Since $R_{c}>0$ from Assumption 3 and $R_{\theta}>0$ from (27), in case (i)

$$
\lim _{\theta \rightarrow \theta^{A}} R(C(\theta), \theta)<R\left(C\left(\theta^{H}\right), \theta^{H}\right) .
$$

Since $h\left(\theta^{A}\right) \in\left(\theta^{A}, \theta^{H}\right), \lim _{\theta \rightarrow \theta^{A}} C(\theta) \leq C\left(h\left(\theta^{A}\right)\right)$ whereas $C\left(\theta^{H}\right)=C\left(h\left(\theta^{H}\right)\right)$ since $h\left(\theta^{H}\right)=\theta^{H}$ from (13). Since applying these properties and (34) to (32) implies $0=$ $\lim _{\theta \rightarrow \theta^{A}} D(\theta)<D\left(\theta^{H}\right)$ in case (i), from (33) we find $C^{\prime}\left(\theta^{H}\right)<0$, which contradicts (i).

In case (ii) $\lim _{\theta \rightarrow \theta^{A}} C(\theta) \leq C\left(\theta^{B}\right)$. Since $R_{c}>0$ from Assumption 3 and $R_{\theta}>0$ from (27), this inequality implies

$$
\lim _{\theta \rightarrow \theta^{A}} R(C(\theta), \theta)<R\left(C\left(\theta^{B}\right), \theta^{B}\right) .
$$

Further, $h\left(\theta^{A}\right)$ is located in either $\left(\theta^{A}, \theta^{B}\right]$ or $\left(\theta^{B}, \theta^{H}\right)$. If $h\left(\theta^{A}\right) \in\left(\theta^{A}, \theta^{B}\right]$, then $C\left(h\left(\theta^{A}\right)\right) \geq \lim _{\theta \rightarrow \theta^{A}} C(\theta)$ since we suppose $C^{\prime}(\theta) \geq 0$ for all $\theta \in\left(\theta^{A}, \theta^{B}\right]$. Contrastingly, $C\left(h\left(\theta^{B}\right)\right) \leq C\left(\theta^{B}\right)$ since $h\left(\theta^{B}\right) \in\left(\theta^{B}, \theta^{H}\right)$ and $C^{\prime}(\theta) \leq 0$ for all $\theta \in\left(\theta^{B}, \theta^{H}\right]$. Using these inequalities, (32) and (35) we find $\lim _{\theta \rightarrow \theta^{A}} D(\theta)<D\left(\theta^{B}\right)$, which contradicts case (ii).

If $h\left(\theta^{A}\right) \in\left(\theta^{B}, \theta^{H}\right)$, then because $h^{\prime}(\theta)>0$ from (13), we find $\theta^{A}<\theta^{B}<h\left(\theta^{A}\right)<$ $h\left(\theta^{B}\right)<\theta^{H}$. In case (ii), this means

$$
\lim _{\theta \rightarrow \theta^{A}} C(\theta) \leq C\left(\theta^{B}\right) \geq C\left(h\left(\theta^{A}\right)\right) \geq C\left(h\left(\theta^{B}\right)\right) .
$$

Thus,

$$
\frac{u^{\prime}\left(C\left(h\left(\theta^{B}\right)\right)\right)}{u^{\prime}\left(C\left(\theta^{B}\right)\right)}-1 \geq \max \left(0, \lim _{\theta \rightarrow \theta^{A}} \frac{u^{\prime}(C(h(\theta)))}{u^{\prime}(C(\theta))}-1\right) .
$$

Applying this property and (35) to (32) yields $\lim _{\theta \rightarrow \theta^{A}} D(\theta)<D\left(\theta^{B}\right)$, which contradicts case (ii). Thus, anyway case (ii) results in a contradiction.

From (32), boundary condition (29) is equivalent to $\lim _{\theta \rightarrow \theta^{*}} D(\theta)=0$. By regarding $\theta^{*}$ as $\theta^{0}$ in Lemma 1 , we find $C(\theta)$ that satisfies (28) and (29) to be strictly downward sloping for all $\theta \in\left(\theta^{*}, \theta^{H}\right]$. 


\section{The existence of upper and lower bounds for $C(\theta)$}

We first show $\bar{c}$ and $\underline{c}$ to be unique and well defined. Since $\theta^{*}<\theta^{H}$ and $R_{\theta}>0$ from (27), under Assumption 2

$$
\lim _{c \rightarrow 0} R\left(\theta^{*}, c\right) \leq \lim _{c \rightarrow 0} R\left(\theta^{H}, c\right)<\rho<\lim _{c \rightarrow \infty} R\left(\theta^{*}, c\right) \leq \lim _{c \rightarrow \infty} R\left(\theta^{H}, c\right) .
$$

Applying this property and Assumption 3 to the intermediate value theorem implies that there are unique and positive $\underline{c}$ and $\bar{c}$ satisfying (30). Furthermore, since $R\left(\theta^{H}, \bar{c}\right)>R\left(\theta^{*}, \bar{c}\right)=\rho=R\left(\theta^{H}, \underline{c}\right)$, Assumption 3 implies $\bar{c}>\underline{c}$.

Next, we prove the following lemma.

Lemma 2 Suppose that $C(\theta)$ satisfies (28) and that there exists $\theta^{0} \in\left[\theta^{*}, \theta^{H}\right)$ satisfying $\lim _{\theta \rightarrow \theta^{0}} D(\theta)=0$. Then, under Assumptions 2 and $3, C(\theta) \in[\underline{c}, \bar{c}]$ for all $\theta \in\left(\theta^{0}, \theta^{H}\right]$.

Proof: As shown by Lemma 1, the last term in (32) is positive when $\theta \rightarrow \theta^{0}$ and hence

$$
\lim _{\theta \rightarrow \theta^{0}} R(\theta, C(\theta))<\rho .
$$

From the first equation of (30), the positivity of $R_{\theta}$ given by (27), and Assumption 3, $R(\theta, c)>\rho$ for all $\theta \in\left[\theta^{*}, \theta^{H}\right]$ and $c>\bar{c}$. Thus, (36) implies $\lim _{\theta \rightarrow \theta^{0}} C(\theta) \leq \bar{c}$.

When $\theta=\theta^{H}$, the last term in (32) equals zero since $h\left(\theta^{H}\right)=\theta^{H}$ from (13). Since $C^{\prime}(\theta)=D(\theta) / g(\theta)$ from (28) and (32), $C^{\prime}\left(\theta^{H}\right) \leq 0$ from Lemma 1, and $g\left(\theta^{H}\right)<0$ from (11), we find $D\left(\theta^{H}\right) \leq 0$. Applying these properties to (32) yields

$$
R\left(\theta^{H}, C\left(\theta^{H}\right)\right) \geq \rho .
$$

Comparing this property with the second equation of (30) and using Assumption 3 yield $C\left(\theta^{H}\right) \geq \underline{c}$. Furthermore, the monotonicity of $C(\theta)$ from Lemma 1 implies $C(\theta) \in\left[C\left(\theta^{H}\right), \lim _{\theta \rightarrow \theta^{0}} C(\theta)\right] \subseteq[\underline{c}, \bar{c}]$ for all $\theta \in\left(\theta^{0}, \theta^{H}\right]$.

Under condition (29), $\theta^{*}$ satisfies the requirement for $\theta^{0}$ in Lemma 2. Thus, $C(\theta) \in[\underline{c}, \bar{c}]$ for all $\theta \in\left(\theta^{*}, \theta^{H}\right]$. 


\section{The uniqueness of $C(\theta)$}

Let $\widetilde{C}\left(\theta, c^{H}\right)$ be the solution to differential equation (28) that satisfies the following boundary condition:

$$
\widetilde{C}\left(\theta^{H}, c^{H}\right)=c^{H}
$$

where $c^{H}(>0)$ is an arbitrary constant. We can solve differential equation (28) backward from $\theta=\theta^{H}$ with boundary condition (37), because $h(\theta)$ is larger than $\theta$ and thus $C(\theta)$ and $C(h(\theta))$ are already known when we calculate the gradient of $C(\cdot)$ at $\theta .{ }^{21}$ Therefore, function $\widetilde{C}\left(\theta, c^{H}\right)$ is uniquely determined within interval $\left(\theta^{*}, \theta^{H}\right]$.

Using function $\widetilde{C}(\cdot)$, boundary condition (29) can be rewritten as

$$
\begin{aligned}
& \lim _{\theta \rightarrow \theta^{*}} \widetilde{D}\left(\theta, c^{H}\right)=0, \text { where } \\
& \widetilde{D}\left(\theta, c^{H}\right) \equiv R\left(\theta, \widetilde{C}\left(\theta, c^{H}\right)\right)-\rho+\theta\left(\frac{u^{\prime}\left(\widetilde{C}\left(h(\theta), c^{H}\right)\right)}{u^{\prime}\left(\widetilde{C}\left(\theta, c^{H}\right)\right)}-1\right)
\end{aligned}
$$

$\widetilde{D}\left(\theta, c^{H}\right)$ is the expression in brackets of $(28)$ with $C(\theta)$ being replaced by $\widetilde{C}\left(\theta, c^{H}\right)$. Functions $\widetilde{C}(\cdot)$ and $\widetilde{D}(\cdot)$ have the following properties: ${ }^{22}$

Lemma 3 Under Assumption 3, (a) $\widetilde{C}_{c}\left(\theta, c^{H}\right)>0$ and (b) $\widetilde{D}_{c}\left(\theta, c^{H}\right)>0$ for all $\theta \in$ $\left(\theta^{*}, \theta^{H}\right]$ and $c^{H}>0$. In addition, (c) there is a constant, $\underline{D_{c}}$, such that $\widetilde{D}_{c}\left(\theta, c^{H}\right)>$ $\underline{D_{c}}>0$ whenever $\widetilde{C}\left(\theta, c^{H}\right) \in[\underline{c}, \bar{c}]$.

Proof: By rearranging terms in (28),

$$
\begin{gathered}
\widetilde{D}\left(\theta, c^{H}\right)=g(\theta) \frac{\gamma\left(\widetilde{C}\left(\theta, c^{H}\right)\right) \widetilde{C}_{\theta}\left(\theta, c^{H}\right)}{\widetilde{C}\left(\theta, c^{H}\right)}=-g(\theta) M_{\theta}\left(\theta, c^{H}\right) \\
\text { where } M\left(\theta, c^{H}\right) \equiv \ln u^{\prime}\left(\widetilde{C}\left(\theta, c^{H}\right)\right) .
\end{gathered}
$$

Differentiating (40) with respect to $c^{H}$ yields

$$
M_{\theta c}\left(\theta, c^{H}\right)=-\widetilde{D}_{c}\left(\theta, c^{H}\right) / g(\theta) .
$$

\footnotetext{
${ }^{21}$ The numerical analysis follows this way. See Appendix B for it.

${ }^{22} \widetilde{C}_{c}\left(\theta, c^{H}\right) \equiv \partial \widetilde{C}\left(\theta, c^{H}\right) / \partial c^{H} . \widetilde{D}_{c}\left(\theta, c^{H}\right)$ and other partial derivatives are defined likewise.
} 
Differentiating (39) with respect to $c^{H}$ gives

$$
\begin{aligned}
\widetilde{D}_{c}\left(\theta, c^{H}\right) & =\Phi\left(\theta, c^{H}\right)+\Psi\left(\theta, c^{H}\right), \text { where } \\
\Phi\left(\theta, c^{H}\right) & \equiv R_{c}\left(\theta, \widetilde{C}\left(\theta, c^{H}\right)\right) \frac{u^{\prime}\left(\widetilde{C}\left(\theta, c^{H}\right)\right)}{u^{\prime \prime}\left(\widetilde{C}\left(\theta, c^{H}\right)\right)} M_{c}\left(\theta, c^{H}\right) \\
\Psi\left(\theta, c^{H}\right) & \equiv \theta \frac{u^{\prime}\left(\widetilde{C}\left(h(\theta), c^{H}\right)\right)}{u^{\prime}\left(\widetilde{C}\left(\theta, c^{H}\right)\right)}\left(M_{c}\left(h(\theta), c^{H}\right)-M_{c}\left(\theta, c^{H}\right)\right) .
\end{aligned}
$$

Since (37) implies $\widetilde{C}_{c}\left(\theta^{H}, c^{H}\right)=1$, differentiating (41) with respect to $c^{H}$ yields

$$
M_{c}\left(\theta^{H}, c^{H}\right)=u^{\prime \prime}\left(c^{H}\right) / u^{\prime}\left(c^{H}\right)<0 .
$$

From (13), $h\left(\theta^{H}\right)=\theta^{H}$ and thus $\Psi\left(\theta^{H}, c^{H}\right)=0$. Using this property, Assumption 3, (37), (43) and (44) we obtain

$$
\widetilde{D}_{c}\left(\theta^{H}, c^{H}\right)=R_{c}\left(\theta^{H}, c^{H}\right)>0
$$

Now we extend this property to all $\theta \in\left(\theta^{*}, \theta^{H}\right]$. To prove this, suppose otherwise. Then, there should be some $\theta^{A} \in\left(\theta^{*}, \theta^{H}\right)$ that satisfies $\widetilde{D}_{c}\left(\theta, c^{H}\right)>0$ for all $\theta \in$ $\left(\theta^{A}, \theta^{H}\right]$ and $\widetilde{D}_{c}\left(\theta^{A}, c^{H}\right) \leq 0$. This property, combined with (42), (44) and the negativity of $g(\theta)$ from (11), gives

$$
\begin{aligned}
M_{c}\left(\theta^{A}, c^{H}\right) & =M_{c}\left(\theta^{H}, c^{H}\right)+\int_{\theta^{A}}^{\theta^{H}} \widetilde{D}_{c}\left(\theta, c^{H}\right) / g(\theta) d \theta \\
& <u^{\prime \prime}\left(c^{H}\right) / u^{\prime}\left(c^{H}\right)<0 .
\end{aligned}
$$

Assumption 3, (43) and (45) imply $\Phi\left(\theta^{A}, c^{H}\right)>0$. Similarly, from (42)

$$
M_{c}\left(h\left(\theta^{A}\right), c^{H}\right)-M_{c}\left(\theta^{A}, c^{H}\right)=-\int_{\theta^{A}}^{h\left(\theta^{A}\right)} \widetilde{D}_{c}\left(\theta, c^{H}\right) / g(\theta) d \theta>0,
$$

which means $\Psi\left(\theta^{A}, c^{H}\right)>0$. Substituting these results into (43) yields $\widetilde{D}_{c}\left(\theta^{A}, c^{H}\right)>$ 0 , which contradicts the assumption that $\widetilde{D}_{c}\left(\theta^{A}, c^{H}\right) \leq 0$. Thus there is no such $\theta^{A}$, and therefore property $(b)$ holds.

Property (b) and (42) imply

$$
M_{\theta c}\left(\theta, c^{H}\right)>0 \text { for all } \theta \in\left(\theta^{*}, \theta^{H}\right] \text { and } c^{H}>0
$$


From (44) and (47),

$$
M_{c}\left(\theta, c^{H}\right)<u^{\prime \prime}\left(c^{H}\right) / u^{\prime}\left(c^{H}\right)<0 \quad \text { for all } \theta \in\left(\theta^{*}, \theta^{H}\right] .
$$

Since (48) is equivalent to $\widetilde{C}_{c}\left(\theta, c^{H}\right)>0$ from (41), property $(a)$ holds.

Finally we prove property $(c)$. Define

$$
\underline{\Phi}(\theta, c) \equiv R_{c}(\theta, c) \frac{u^{\prime}(c) u^{\prime \prime}\left(c^{H}\right)}{u^{\prime \prime}(c) u^{\prime}\left(c^{H}\right)}
$$

From Assumption 3, $\underline{\Phi}(\theta, c)$ is positive and continuous for all $(\theta, c) \in \Theta \equiv\left[\theta^{*}, \theta^{H}\right] \times$ $[\underline{c}, \bar{c}]$. Since $\Theta$ is a compact set, there exists $\underline{D_{c}} \equiv \min _{(\theta, c) \in \Theta} \underline{\Phi}(\theta, c)>0$. Combined with (43), (47) and (48), this property implies

$$
\widetilde{D}_{c}\left(\theta, c^{H}\right)>\Phi\left(\theta, c^{H}\right)>R_{c}\left(\theta, \widetilde{C}\left(\theta, c^{H}\right)\right) \frac{u^{\prime}\left(\widetilde{C}\left(\theta, c^{H}\right)\right) u^{\prime \prime}\left(c^{H}\right)}{u^{\prime \prime}\left(\widetilde{C}\left(\theta, c^{H}\right)\right) u^{\prime}\left(c^{H}\right)} \geq \underline{D_{c}}
$$

whenever $\widetilde{C}\left(\theta, c^{H}\right) \in[\underline{c}, \bar{c}]$.

We now prove the uniqueness of function $C(\theta)$ using Lemmata 2 and 3. Suppose that there are two distinct functions $C_{1}(\theta)$ and $C_{2}(\theta)$ both of which satisfy (28) and (29). Let $c_{1}^{H} \equiv C_{1}\left(\theta^{H}\right)$ and $c_{2}^{H} \equiv C_{2}\left(\theta^{H}\right)$. Then $C_{1}(\theta)=\widetilde{C}\left(\theta, c_{1}^{H}\right)$ and $C_{2}(\theta)=$ $\widetilde{C}\left(\theta, c_{2}^{H}\right)$ for all $\theta \in\left(\theta^{*}, \theta^{H}\right]$. Note that $c_{1}^{H} \neq c_{2}^{H}$ because we have assumed that $C_{1}(\theta)$ and $C_{2}(\theta)$ are distinct functions. Since (29) is equivalent to (38), both functions satisfy

$$
\lim _{\theta \rightarrow \theta^{*}} \widetilde{D}\left(\theta, c_{1}^{H}\right)=\lim _{\theta \rightarrow \theta^{*}} \widetilde{D}\left(\theta, c_{2}^{H}\right)=0 .
$$

From Lemma 2, $\widetilde{C}\left(\theta, c_{1}^{H}\right), \widetilde{C}\left(\theta, c_{2}^{H}\right) \in[\underline{c}, \bar{c}]$ for all $\theta \in\left(\theta^{*}, \theta^{H}\right]$. Applying it to property $(a)$ of Lemma 3 implies $\widetilde{C}\left(\theta, c^{H}\right) \in[\underline{c}, \bar{c}]$ for all $c^{H} \in\left[c_{1}^{H}, c_{2}^{H}\right]$ and all $\theta \in$ $\left(\theta^{*}, \theta^{H}\right]$. Thus, we can use property $(c)$ of Lemma 3 to obtain

$$
\lim _{\theta \rightarrow \theta^{*}}\left|\widetilde{D}\left(\theta, c_{1}^{H}\right)-\widetilde{D}\left(\theta, c_{2}^{H}\right)\right|>\left|c_{1}^{H}-c_{2}^{H}\right| \underline{D_{c}}>0
$$

which contradicts (49). 


\section{The existence of $C(\theta)$}

We obtain another property with respect to $\widetilde{D}\left(\theta, c^{H}\right)$.

Lemma 4 Under Assumptions 2 and 3, (a) $\widetilde{D}(\theta, \underline{c})<0$ and (b) $\widetilde{D}(\theta, \bar{c})>0$ for all $\theta \in\left(\theta^{*}, \theta^{H}\right)$.

Proof: We first prove property (a). From (27) and (30),

$$
R\left(\theta^{H}, \underline{c}\right)=\rho \quad \text { and } \quad R(\theta, \underline{c})<\rho \text { for all } \theta \in\left(\theta^{*}, \theta^{H}\right)
$$

Since (13) implies $h\left(\theta^{H}\right)=\theta^{H}$, substituting (37) and (51) into (39) yields

$$
\widetilde{D}\left(\theta^{H}, \underline{c}\right)=0 \text {. }
$$

Since (40) and (52) imply $\widetilde{C}_{\theta}\left(\theta^{H}, \underline{c}\right)=0$, and $R_{\theta}>0$ from (27), differentiating (39) with respect to $\theta$ when $\theta=\theta^{H}$ gives

$$
\widetilde{D}_{\theta}\left(\theta^{H}, \underline{c}\right)=R_{\theta}\left(\theta^{H}, \underline{c}\right)>0
$$

Equations (52) and (53) show that there is a small $\varepsilon(>0)$ such that $\widetilde{D}(\theta, \underline{c})<0$ for all $\theta \in\left(\theta^{H}-\varepsilon, \theta^{H}\right)$.

We now extend the negativity of $\widetilde{D}(\theta, \underline{c})$ to the whole interval of $\left(\theta^{*}, \theta^{H}\right)$. To see this, suppose otherwise. Then, there must be some $\theta^{A} \in\left(\theta^{*}, \theta^{H}\right)$ such that $\widetilde{D}(\theta, \underline{c})<$ 0 for all $\theta \in\left(\theta^{A}, \theta^{H}\right)$ and that $\widetilde{D}\left(\theta^{A}, \underline{c}\right) \geq 0$. Since $\widetilde{D}<0 \Leftrightarrow \widetilde{C}_{\theta}>0$ from (40) and the negativity of $g(\theta)$ in (11), we obtain $\widetilde{C}\left(\theta^{A}, \underline{c}\right)<\widetilde{C}\left(h\left(\theta^{A}\right), \underline{c}\right)<\widetilde{C}\left(\theta^{H}, \underline{c}\right)=\underline{c}$. With Assumption 3 and (51), these inequalities yield $R\left(\theta^{A}, \widetilde{C}\left(\theta^{A}, \underline{c}\right)\right)<R\left(\theta^{A}, \underline{c}\right)<\rho$ and hence from $(39) \widetilde{D}\left(\theta^{A}, \underline{c}\right)<0$, which is a contradiction.

Next we prove property (b). From Assumptions 2 and 3,

$$
R(\theta, \bar{c})>\rho \text { for all } \theta \in\left(\theta^{*}, \theta^{H}\right]
$$

It implies $\widetilde{D}\left(\theta^{H}, \bar{c}\right)>0$ from (39) since $h\left(\theta^{H}\right)=\theta^{H}$ from (13). To prove that this inequality actually holds for whole $\left(\theta^{*}, \theta^{H}\right]$, suppose otherwise. Then, there should be 
some $\theta^{A} \in\left(\theta^{*}, \theta^{H}\right)$ such that $\widetilde{D}(\theta, \bar{c})>0$ for all $\theta \in\left(\theta^{A}, \theta^{H}\right]$ and that $\widetilde{D}\left(\theta^{A}, \bar{c}\right) \leq 0$. From (33), we find $\widetilde{C}\left(\theta^{A}, \bar{c}\right)>\widetilde{C}\left(h\left(\theta^{A}\right), \bar{c}\right)>\widetilde{C}\left(\theta^{H}, \bar{c}\right)=\bar{c}$. With Assumption 3 and (54), these inequalities imply $R\left(\theta^{A}, \widetilde{C}\left(\theta^{A}, \bar{c}\right)\right)>R\left(\theta^{A}, \bar{c}\right)>\rho$ and therefore from (39) $\widetilde{D}\left(\theta^{A}, \bar{c}\right)>0$, which is again a contradiction.

Applying property (b) of Lemma 3 and Lemma 4 to the intermediate value theorem assures that, for any given $\theta^{0} \in\left(\theta^{*}, \theta^{H}\right)$, there uniquely exists $c^{H} \in[\underline{c}, \bar{c}]$ that satisfies $\widetilde{D}\left(\theta^{0}, c^{H}\right)=0$. That is, there is a unique function, $c^{H}=\zeta\left(\theta^{0}\right)$, satisfying

$$
\widetilde{D}\left(\theta^{0}, \zeta\left(\theta^{0}\right)\right)=0 \text { for all } \theta^{0} \in\left(\theta^{*}, \theta^{H}\right)
$$

From Lemmata 1 and 2, function $\widetilde{C}\left(\theta, \zeta\left(\theta^{0}\right)\right)$ is monotonic and bounded by $[\underline{c}, \bar{c}]$ within interval $\theta \in\left(\theta^{0}, \theta^{H}\right]$. By taking limit as $\theta^{0} \rightarrow \theta^{*}$, we conclude that $C(\theta)=$ $\widetilde{C}\left(\theta, c^{H *}\right)$ is monotonic and bounded for all $\theta \in\left(\theta^{*}, \theta^{H}\right]$, where $c^{H *} \equiv \lim _{\theta^{0} \rightarrow \theta^{*}} \zeta\left(\theta^{0}\right) .{ }^{23}$ This implies $c_{t}$ should neither explode, implode nor oscillate. Thus $\dot{c}_{t} / c_{t} \rightarrow 0$ as $\theta_{t}^{e} \rightarrow \theta^{*}$, which gives the validity of boundary condition (29).

\section{Appendix B: Numerical procedure of finding $C(\cdot)$}

Our problem is generally called an IVP (initial value problem), which is usually solved by finite difference methods, such as the Runge-Kutta method and the Euler method. ${ }^{24}$ However, we cannot use them since the right-hand side of (28) contains $C(h(\theta))$, which makes impossible to calculate the gradient of $C(\theta)$ before $C(h(\theta))$ is determined. Since $h(\theta)$ is always larger than $\theta$, we cannot solve the differential equation forward from $\theta^{*}$, where the boundary condition is given, toward $\theta^{H}$. Instead,

\footnotetext{
${ }^{23}$ Note that, $\zeta(\theta)$ is bounded by $\underline{c}$ and $\bar{c}$ from Lemma 2 . In addition, by totally differentiating (55) and utilizing Lemma 1 , we can prove that $\zeta(\theta)$ is continuous and monotonic for all $\theta \in\left(\theta^{*}, \theta^{H}\right]$. It means that $\zeta\left(\theta^{0}\right)$ does not oscillate as $\theta^{0} \rightarrow \theta^{*}$, hence the existence of $c^{H *} \equiv \lim _{\theta^{0} \rightarrow \theta^{*}} \zeta\left(\theta^{0}\right)$ is guaranteed.

${ }^{24}$ See, for example, Judd (1998).
} 
we can solve it backward from $\theta^{H}$ toward $\theta^{*}$, during which $C(h(\theta))$ is already known when we calculate the gradient of $C(\theta)$.

This strategy, however, involves another difficulty because the value of the function at the starting point, $c^{H} \equiv C\left(\theta^{H}\right)$, is not predetermined. Thus, we have to find an appropriate initial value $c^{H}$ such that boundary condition (29) is eventually met when (28) is solved from it. ${ }^{25}$ This method is in fact used when we prove proposition 1 in appendix $\mathrm{A}-$ i.e., we show that there is a unique $c^{H *} \in[\underline{c}, \bar{c}]$ that satisfies this property. In the numerical analysis we calculate $c^{H *}$ in the following way:

Step 1. Let $i=0, h_{0}=\bar{c}$ and $l_{0}=\underline{c}$.

Step 2. Let $c_{i}^{H}=\left(h_{i}+l_{i}\right) / 2$. Using the Euler method, solve differential equation (28) (to which (31) is applied) backward starting from boundary value $C\left(\theta^{H}\right)=c_{i}^{H}$.

Step 3. If $C(\theta)$ exceeds $\bar{c}$ during the calculation, or if $D(\theta)$ defined by (32) remains positive when $\theta$ approaches $\theta^{*}$, let $h_{i+1}=c_{i}^{H}$ and $l_{i+1}=l_{i}$. Conversely, if $C^{\prime}(\theta)$ becomes positive during the calculation or if $D(\theta)$ remains negative when $\theta$ approaches $\theta^{*}$, let $h_{i+1}=h_{i}$ and $l_{i+1}=c_{i}^{H}$. Otherwise, $c_{i}^{H}$ is the solution.

Step 4. Let $i=i+1$.

Step 5. Repeat steps 2-4 until $h_{i}$ and $l_{i}$ get sufficiently close to each other. Then admit $c^{H}=\left(h_{i}+l_{i}\right) / 2$ as the solution.

\section{References}

[1] Andolfatto, David and Paul Gomme (2003), "Monetary Policy Regimes and Beliefs," International Economic Review, 44(1), 1-30.

[2] Chalkley, Martin and In Ho Lee (1997), "Learning and Asymmetric Business Cycles," Review of Economic Dynamics, 1, 623-645.

\footnotetext{
${ }^{25}$ This method is usually called 'monkey hunting'.
} 
[3] Driffill, John and Marcus Miller (1993), "Learning and Inflation Convergence in the ERM," Economic Journal, 103, 369-378.

[4] Hamilton, James D. (1989), "A New Approach to the Economic Analysis of Nonstationary Time Series and the Business Cycle," Econometrica, 57(2), 357384.

[5] Judd, Kenneth L. (1998), Numerical Methods in Economics, The MIT Press.

[6] Lucas, Robert E. Jr. (1978), "Asset Prices in an Exchange Economy," Econometrica, 46(6), 1429-1445.

[7] Ono, Yoshiyasu (1994), Money, Interest, and Stagnation, Oxford University Press.

[8] Ono, Yoshiyasu (2001), “ A Reinterpretation of Chapter 17 of Keynes's General Theory: Effective Demand Shortage Under Dynamic Optimization," International Economic Review, 42, 207-236.

[9] Potter, Simon M. (2000), "A Nonlinear Model of Business Cycle," Studies in Nonlinear Dynamics and Econometrics, 4(2), 85-93.

[10] Sidrauski, Miguel (1967), "Rational Choice and Pattern of Growth in a Monetary Economy," American Economic Review, 57(2), 534-544.

[11] Sill, Keith and Jeff Wrase (1999), "Exchange Rates, Monetary Policy Regimes, and Beliefs," Federal Reserve Bank of Philadelphia Working Paper, 99-6.

[12] Zeira, Joseph (1999), "Informational Overshooting, Booms, and Crashes," Journal of Monetary Economics, 43, 237-257. 


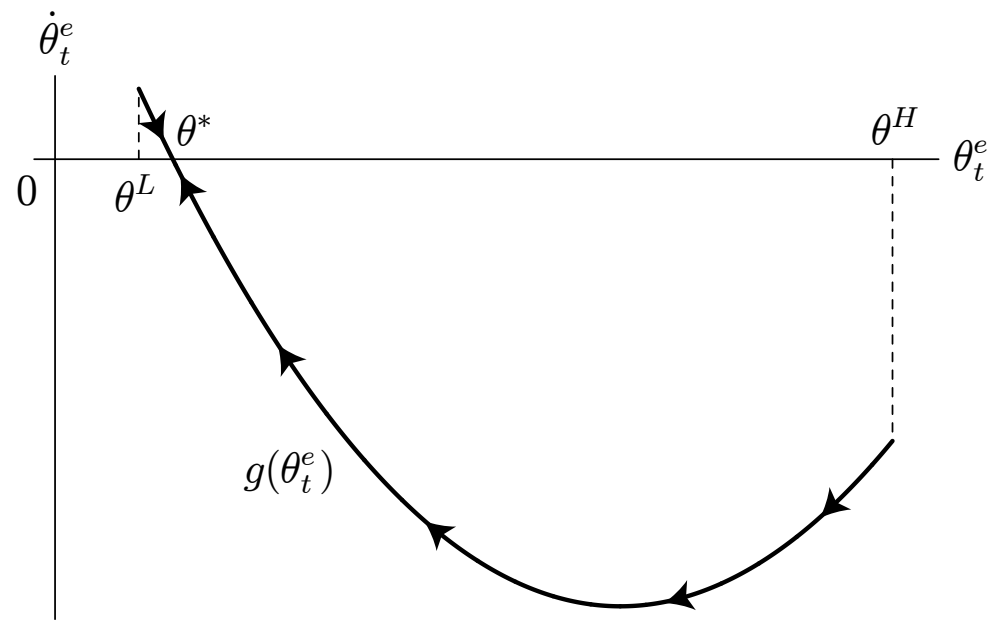

Figure 1: Movement of $\theta_{t}^{e}$ in absence of the shock 


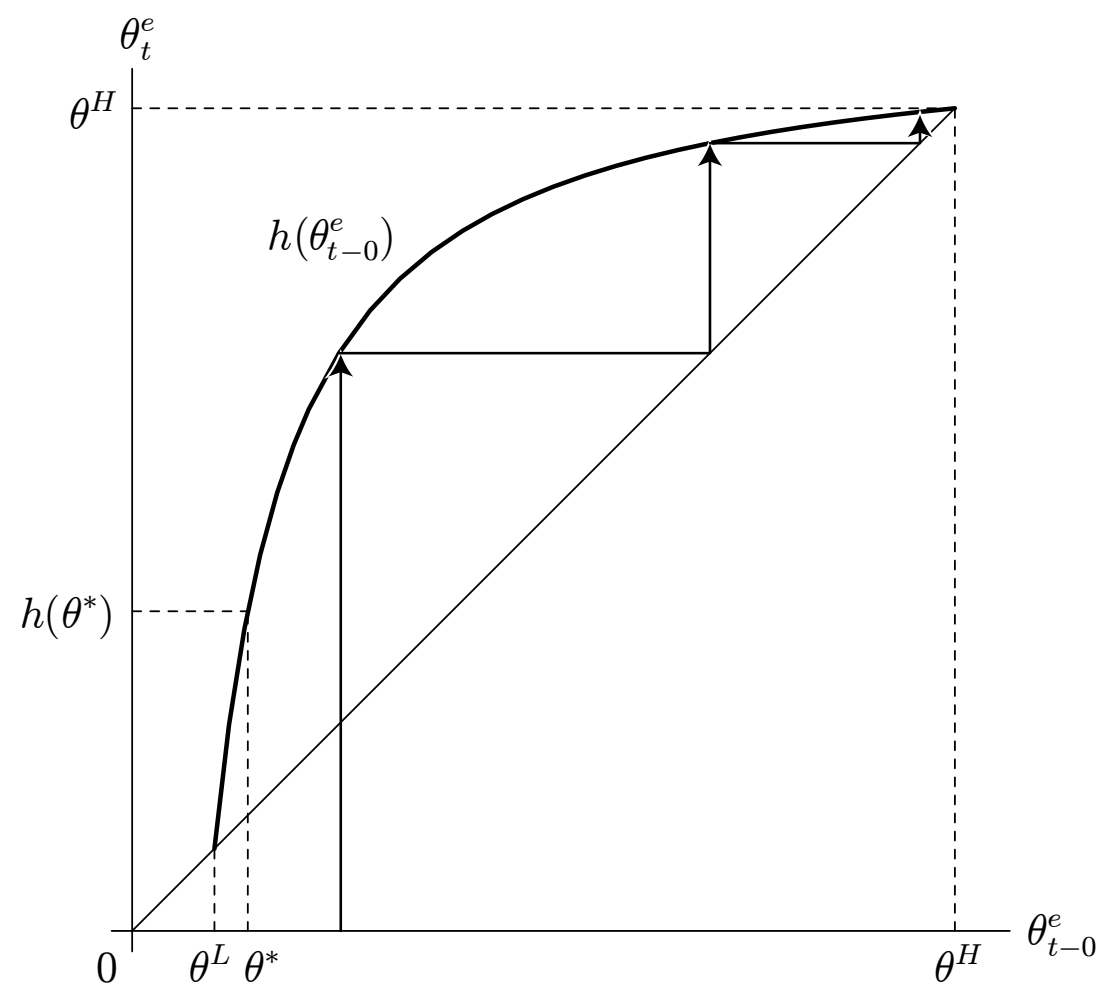

Figure 2: Movement of $\theta_{t}^{e}$ when the shock occurs 


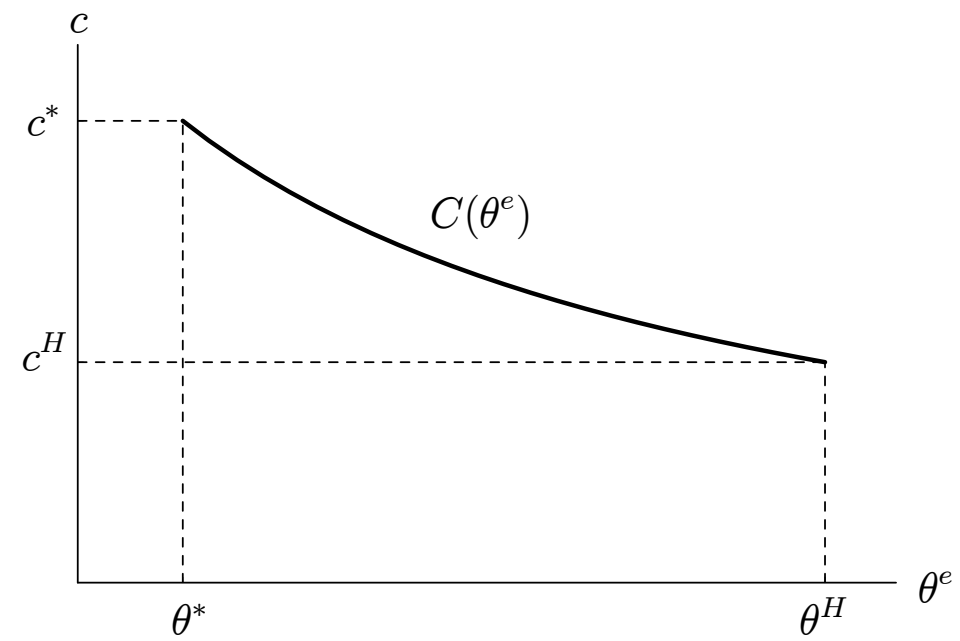

Figure 3: The shape of function $C\left(\theta_{t}^{e}\right)$ 


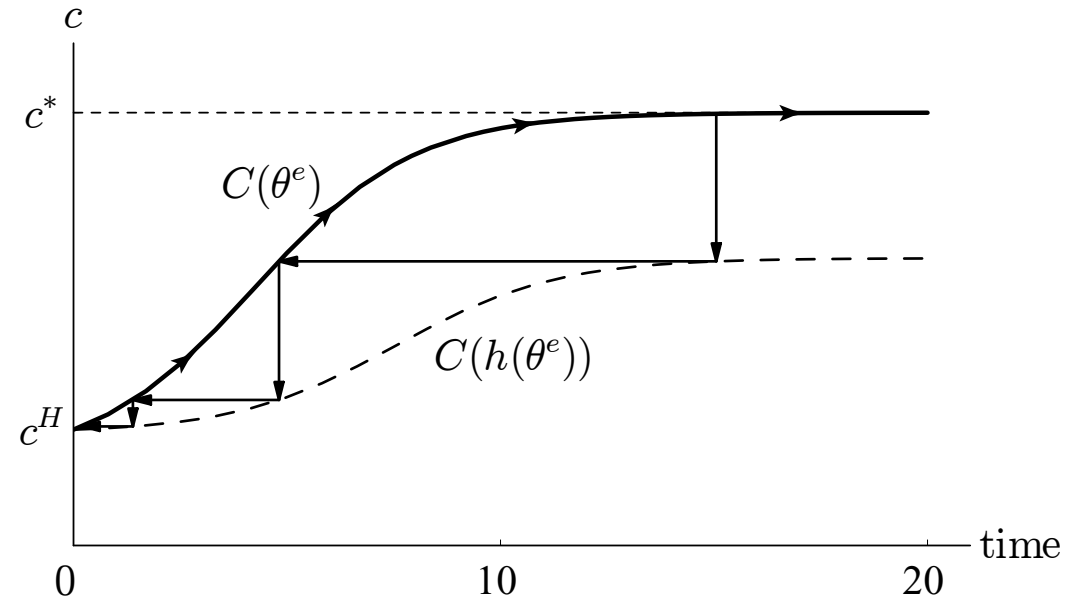

Figure 4: Dynamics of consumption 

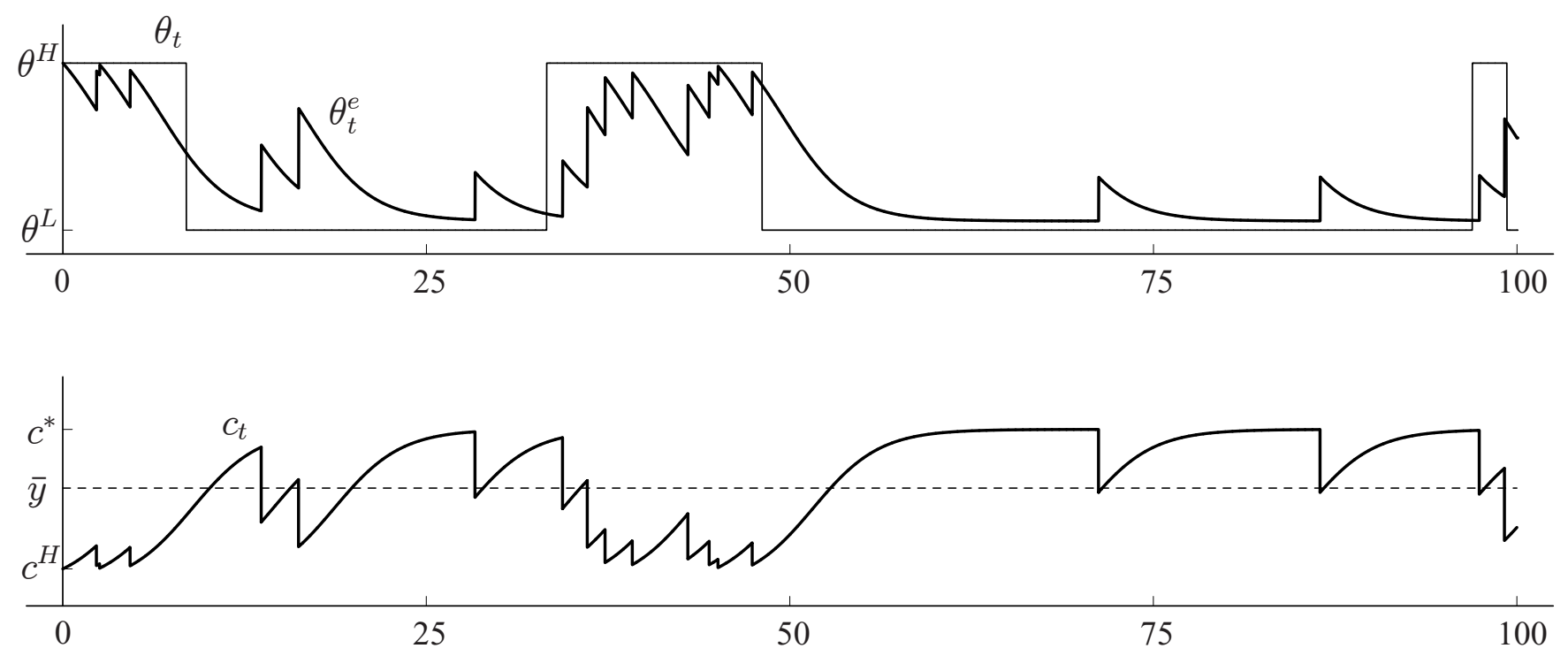

Figure 5: Realized paths of $\theta_{t}, \theta_{t}^{e}$ and $c_{t}$ 